\title{
Virus-induced tumor inflammation facilitates effective DC cancer immunotherapy in a Treg-dependent manner in mice
}

\author{
Norman Woller, ${ }^{1}$ Sarah Knocke, ${ }^{1}$ Bettina Mundt, ${ }^{1}$ Engin Gürlevik, ${ }^{1}$ Nina Strüver, ${ }^{1}$ Arnold Kloos, ${ }^{1}$ \\ Bita Boozari, ${ }^{1}$ Peter Schache, ${ }^{1}$ Michael P. Manns, ${ }^{1}$ Nisar P. Malek, ${ }^{1}$ Tim Sparwasser, ${ }^{2}$ \\ Lars Zender, ${ }^{1,3}$ Thomas C. Wirth, ${ }^{1}$ Stefan Kubicka, ${ }^{1,4}$ and Florian Kühnel ${ }^{1}$ \\ 'Department of Gastroenterology, Hepatology and Endocrinology, Hannover Medical School, Hannover, Germany. ${ }^{2}$ Institute of Infection Immunology,
TWINCORE, Centre for Experimental and Clinical Infection Research, Hannover, Germany. ${ }^{3}$ Helmholtz Centre for Infection Research (HZI), \\ Braunschweig, Germany. ${ }^{4}$ Kreiskliniken Reutlingen, Reutlingen, Germany.
}

\begin{abstract}
Vaccination using DCs pulsed with tumor lysates or specific tumor-associated peptides has so far yielded limited clinical success for cancer treatment, due mainly to the low immunogenicity of tumor-associated antigens. In this study, we have identified intratumoral virus-induced inflammation as a precondition for effective antitumor DC vaccination in mice. Administration of a tumor-targeted DC vaccine during ongoing virus-induced tumor inflammation, a regimen referred to as oncolysis-assisted DC vaccination (ODC), elicited potent antitumoral $\mathrm{CD8}^{+} \mathrm{T}$ cell responses. This potent effect was not replicated by TLR activation outside the context of viral infection. ODC-elicited immune responses mediated marked tumor regression and successful eradication of preestablished lung colonies, an essential prerequisite for potentially treating metastatic cancers. Unexpectedly, depletion of Tregs during ODC did not enhance therapeutic efficacy; rather, it abrogated antitumor cytotoxicity. This phenomenon could be attributed to a compensatory induction of myeloid-derived suppressor cells in Treg-depleted and thus vigorously inflamed tumors, which prevented ODC-mediated immune responses. Consequently, Tregs are not only general suppressors of immune responses, but are essential for the therapeutic success of multimodal and temporally fine-adjusted vaccination strategies. Our results highlight tumor-targeting, replication-competent viruses as attractive tools for eliciting effective antitumor responses upon DC vaccination.
\end{abstract}

\section{Introduction}

Vaccinations using DCs pulsed with tumor lysates or specific tumor-associated peptides are capable of eliciting cytotoxic $\mathrm{T}$ cell responses against tumor antigens. However, DC immunotherapies led to unsatisfying therapeutic results against established tumors in animal models and clinical trials (1-3). The limited efficacy of DC vaccines in cancer immunotherapy appears to be mainly attributable to tumor-specific immunotolerance. In contrast to the low immunogenicity of tumor-associated antigens (TAAs), viruses stimulate evolutionarily conserved cellular danger pathways, triggering strong innate and adaptive immune responses. It has been shown that induction of immune responses upon viral infections depends, at least in part, on recognition of viral DNA and RNA by host pattern-recognition receptors (4). Other groups have reported that viral infections induce immune responses not only against viral antigens, but also against cross-presented cellular antigens in a MyD88- and/or TLR-dependent manner (5, 6). Accordingly, crosspresentation of cellular antigens during viral infections provides a rationale for induction of antitumoral immune responses in tumor treatment strategies using tumor-specific oncolytic viruses.

During viral infection, the immune system evaluates virus-specific and infection-associated danger signals for the fine adjustment of immune responses to a level that keeps infection under control but prevents severe tissue damage $(7,8)$. Recently, it has

Authorship note: Stefan Kubicka and Florian Kühnel contributed equally to this work. Conflict of interest: The authors have declared that no conflict of interest exists. Citation for this article: JClin Invest. 2011;121(7):2570-2582. doi:10.1172/JCI45585. been described that $\mathrm{CD} 4^{+} \mathrm{CD} 25^{+} \mathrm{Foxp}^{+}$Tregs play an essential role in this scenario by calibrating the intensity of inflammation in virus-infected tissue $(9,10)$. Depending on the situation, the presence of Tregs can be either beneficial or detrimental for the host. Tregs can prevent collateral tissue damage $(11,12)$ and autoimmunity (13) during viral infections, but it has also been shown that Tregs can suppress antiviral immune responses, thus facilitating chronic viral diseases $(14,15)$. Corresponding studies with animal infection models showed that anti-CD25 Ab-mediated depletion of Tregs supported systemic antiviral $\mathrm{CD}^{+} \mathrm{T}$ cell responses and subsequent clearance of the pathogen (16). In contrast to these immunosuppressive features, it has been demonstrated that Tregs play an important role in early protective responses to local virus infections by allowing the immediate access of immune cells to infected tissue (17). These apparently opposing features of Tregs indicate distinct roles of Tregs in local and systemic virus infections or at different stages of the immune response.

In the present study, we found that coordinated application of a tumor-directed DC vaccine in the context of virus-induced tumor inflammation elicited a potent antitumoral CD8 immune response. The concurrent inhibition of virus-specific Igs after oncolysis-assisted DC vaccination (ODC; referring to DC vaccination at the time of viral tumor inflammation) suggests an interference of antiviral and antitumoral immune responses. ODC facilitated effective regression of primary tumors and elimination of lung colonies. Surprisingly, the interference of virus/tumor immune responses relied on the presence of Tregs. Treg depletion led to a compensatory induction of myeloid-derived suppressor 
A
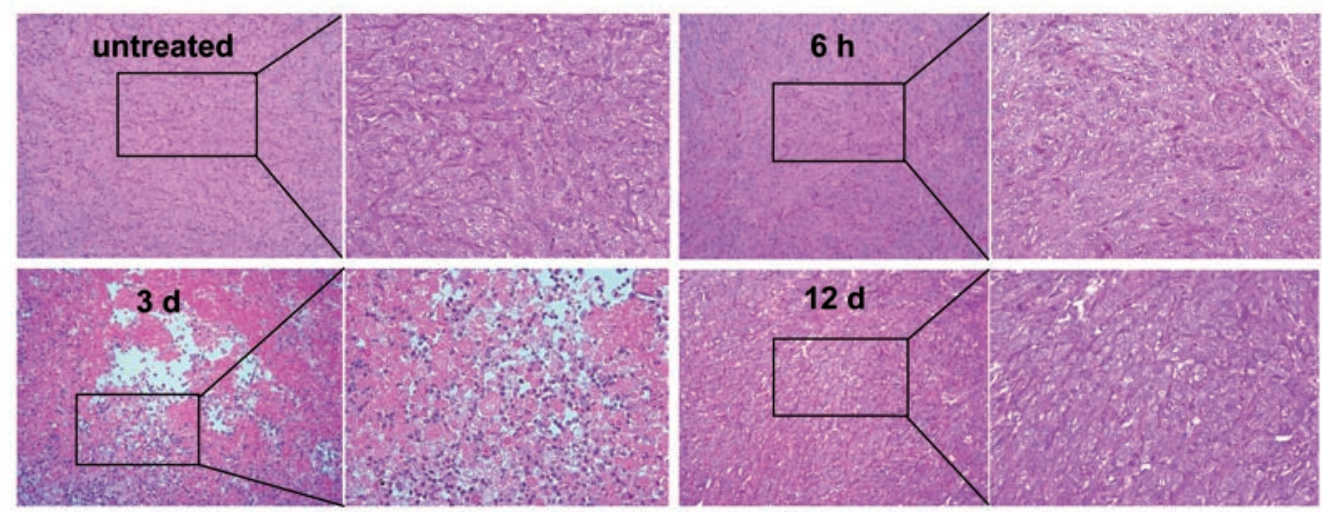

B
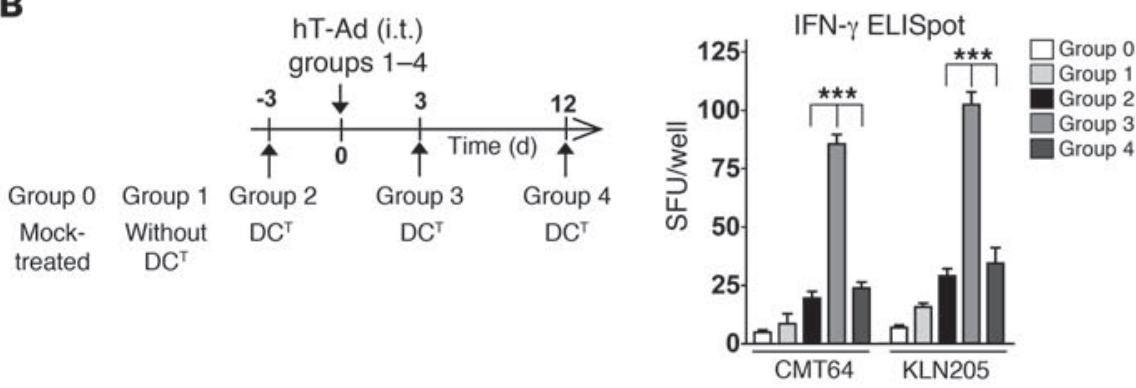

C

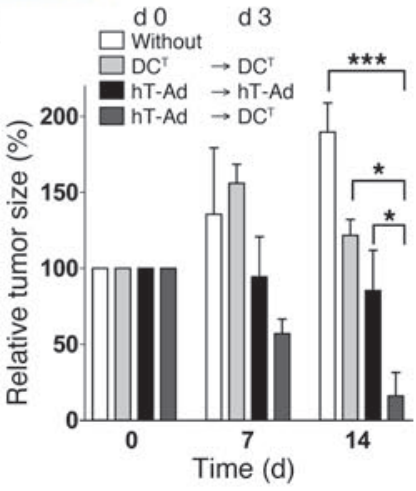

\section{Figure 1}

DC vaccination during viral tumor inflammation elicits a potent and therapeutically efficient antitumor immune response. (A) s.c. grown KLN205 tumors in DBA/2 mice were inoculated with i.t. injection of hTert-Ad. Virus-mediated inflammation of tumor tissue was histologically examined on H\&E-stained slides at the indicated times. Original magnification, $\times 100$ (low-power) and $\times 200$ (high-power). (B) s.c. grown KLN205 or CMT64 tumors were treated with hTert-Ad (hT-Ad) i.t. and tumor cell lysate-pulsed DCs (DC ${ }^{\mathrm{T}}$ ), as shown in the schematic. 14 days after initial treatment, splenocytes of mice were harvested, and ELISpot assays were performed after restimulation with tumor cell lysate. Number of tumor-specific splenocytes in a representative experiment is shown as spot-forming units (SFU) per well ( $n=5$ per group, 4 independent experiments). (C) s.C. grown KLN205 tumors were treated with hTert-Ad i.t. followed by tumor cell lysate-pulsed DCs on day 3 after viral treatment. This treatment was compared with mice receiving 2 subsequent i.t. injections of virus or 2 subsequent $D C$ vaccinations. All treatments were performed on days 0 and 3. Untreated tumor-bearing mice served as control ( $n=8$ per group, 2 independent experiments). ${ }^{*} P \leq 0.05 ;{ }^{* * *} P<0.001$.

cells (MDSCs) in the infected tumor environment that suppressed DC vaccination and abolished effective tumor regression of lung colonies after ODC. Our results indicate that the temporal fine adjustment of immune responses by ODC rather than an imbalanced vigorous tumor inflammation enables therapeutic success of immunotherapy in metastatic cancers.

\section{Results}

Virus-induced inflammation of an established tumor allows for effective antitumoral DC vaccination. Our first aim was to establish syngeneic, immunocompetent murine tumor models to investigate a potential therapeutic benefit for tumor-directed DC vaccinations when combined with intratumoral (i.t.) application of the tumor-selectively replicating Ad hTert-Ad (18). It is known that replication of Ad DNA in murine cells can induce apparent cytolysis without generation of infectious viral particles $(19,20)$. However, we and others have described that some murine cell lines, in particular KLN205 and CMT64, support the complete replication cycle of human Ad with reduced efficacy (21-23). To test the applicability of these cells for a murine model of viral tumor inflammation, we characterized in more detail DNA replication and production of infectious progeny of hTert-Ad in vitro and in vivo. We detected robust Ad DNA replication in both KLN205 and CMT64 cells in vitro (Supplemental Figure 1A; supplemental material available online with this article; doi:10.1172/JCI45585DS1). At 48 hours after i.t. virus injection in s.c. engrafted syngeneic KLN205 and CMT64 tumors, we found up to 100-fold more DNA of hTert-Ad compared with a replication-deficient Ad (rdAd; Supplemental Figure 1B). Assessing the production of infectious viral particles per cell in vitro, we observed low production of infectious progeny by CMT64 cells, but significant virus production in KLN205 cells, almost comparable to levels that can be found in moderately productive human tumor cell lines (Supplemental Figure 1C). Consistently, de novo generation of infectious particles of hTert-Ad was also confirmed in vivo (Supplemental Figure 1D). Although the extent of ongoing replication might not fully reflect Ad-mediated oncolysis in human tumors, these results suggest that hTertAd-infected KLN205 and CMT64 tumors can be regarded as oncolytic models. Furthermore, different abilities of these cells to form infectious particles provided a tool to dissect the roles of viral DNA replication and production of infectious progeny in our study.

Next, we histologically analyzed the extent of the inflammation in s.c. grown KLN205 tumors on syngeneic, immunocompetent $\mathrm{DBA} / 2$ mice in a time course after i.t. injection of hTert-Ad. As 

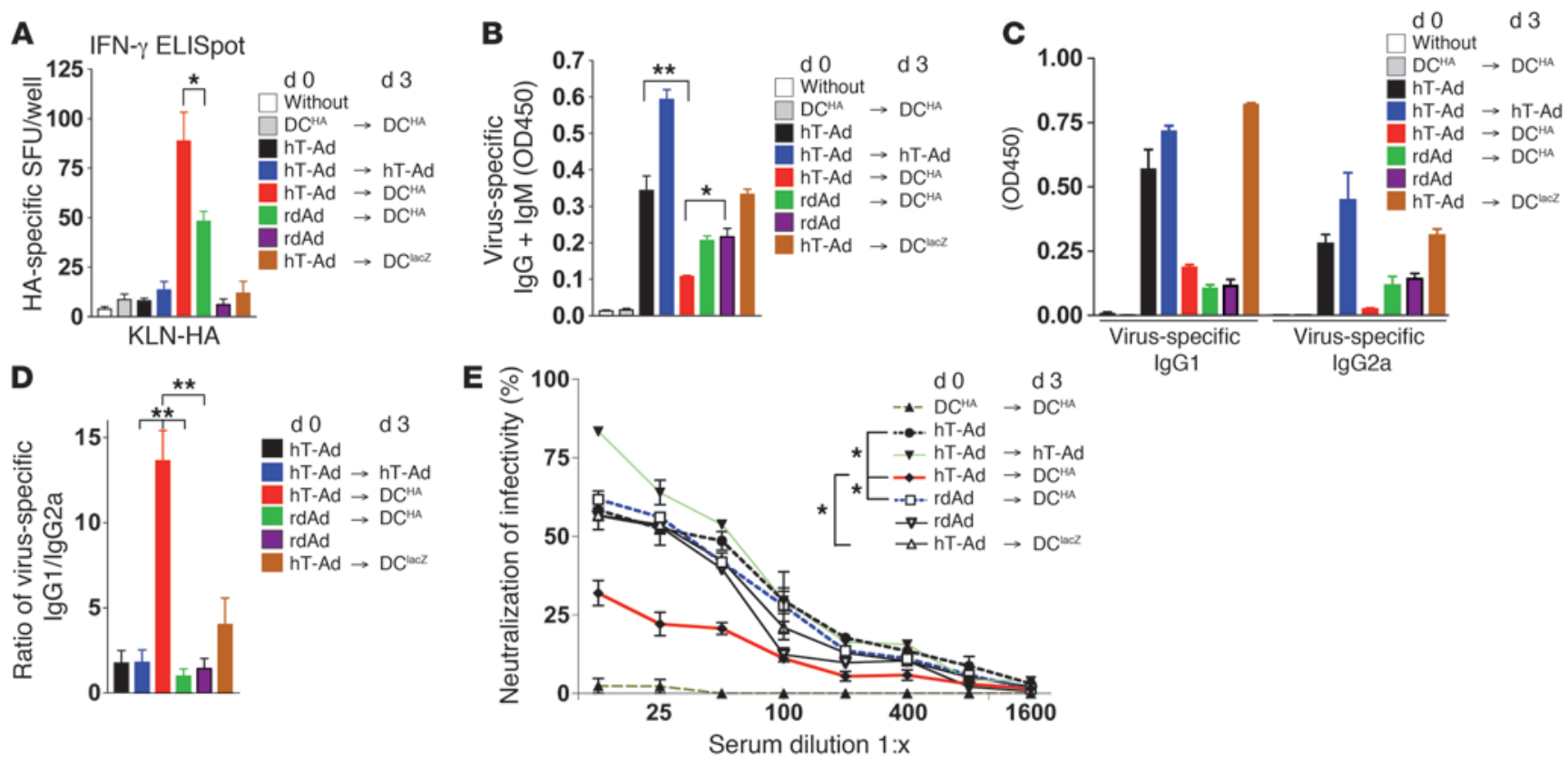

Figure 2

DC vaccination assisted by viral tumor inflammation primes a potent cellular immune response against the tumor model antigen $\mathrm{HA}$ and modifies the virus-specific Ig pattern. (A) KLN-HA cells were injected into the flanks of DBA/2 mice for tumor induction. KLN-HA tumors were treated with hTert-Ad i.t. and/or HA-pulsed DCs (DCHA). After 3 days, mice received a second treatment as indicated. Application of rdAd or DCs pulsed with irrelevant antigen (DClacz) served as controls. At day 14 after the first treatment, splenocytes were harvested, and HA-specific T cell responses were determined by IFN- $\gamma$ ELISpot ( $n=4$ per group, 2 independent experiments). (B) At the same time, blood samples were drawn from treated mice, and serum was investigated for virus-specific Igs by ELISA. Total IgG and IgM responses against the viral capsid are shown. (C) Contribution of IgG1 and IgG2a isotypes to the virus-specific humoral response, determined by ELISA. (D) The IgG1/IgG2a ratio was calculated to estimate the Th1/Th2 character of the humoral response. (E) The neutralizing activity of the virus-specific humoral response was determined in serial dilutions. All values are normalized against serum of naive mice. ${ }^{*} P \leq 0.05 ;{ }^{* \star} P<0.01$.

shown in Figure 1A, viral tumor infection led to efficient lysis of cancer cells and impaired integrity of tumor tissue accompanied by strong lymphocytic infiltration into the inflamed nodule. The maximum viral tumor inflammation was observed 3 days after tumor infection. Interestingly, we observed a dramatic loss of viral DNA in hTert-Ad-infected tumors between 48 and 72 hours after infection (Supplemental Figure 1B), which may reflect the rapid clearance of virus-infected tumor cell debris by tumor-infiltrating lymphocytes. Tumor inflammation then declined, and tissue integrity was completely restored (Figure 1A). No signs of lytic tumor destruction or immune cell infiltration were observable at day 12. Similar results were obtained in a second model using s.c. CMT64 tumors (data not shown). We then investigated whether existing virus-induced inflammation of an established tumor is capable of supporting the effect of tumor-directed DC vaccination. Corresponding to the schematic presentation in Figure 1B, we investigated whether antitumoral DC vaccination can elicit a cellular antitumor response when given at different time points relative to viral tumor inflammation. For the preparation of the DC vaccine, DCs were loaded with whole cell lysate from KLN205 and CMT64 cells. Tumor-specific splenocytes were detected in ELISpot assays after stimulation with the corresponding lysates. Virus-mediated tumor inflammation and additional DC vaccination before or after tumor inflammation elicited only weak antitumoral immune responses (Figure 1B). When DC vaccination was performed at the time of maximum inflammation of the tumor tissue (at day 3 ), we observed a strongly enhanced antitumoral immune response. Then we examined the therapeutic efficacy of DC vaccination at the time of viral tumor inflammation (i.e., ODC). The term was chosen because of the lytic phenotype of the virus-affected tissue (Figure $1 \mathrm{~A})$.

Tumor growth inhibition after ODC was then compared with that after 2 regimens of virotherapy or 2 regimens of DC vaccination. In ODC-treated mice, we observed a considerably reduced tumor burden (Figure 1C). Remarkably, complete tumor remissions leading to long-term survival were found only in the ODC group.

Antitumoral $C D 8^{+} T$ cell immune responses after ODC interfere with the development of virus-specific humoral immunity. Our data suggested that the enhanced tumor-directed cellular response induced by ODC could indirectly affect the development of antiviral immunity. First, we determined the effect of replication on antiviral immune responses. Compared with rdAd, replication of hTert-Ad in KLN205 and CMT64 cells in vivo resulted in significantly enhanced production of antiviral Abs and CD8 immune responses against the viral hexon protein (Supplemental Figure 2).

For a more detailed analysis of ODC-induced immune responses, we stably expressed the model antigens HA in KLN205 cells and OVA in CMT64 cells (referred to herein as the KLN-HA model and the CMT-OVA model, respectively) to allow for epitope-specific evaluation of antitumoral $\mathrm{CD}^{+} \mathrm{T}$ cell responses. In agreement with the results from experiments with tumor lysate-pulsed DCs, ODC using HA-pulsed DCs strongly enhanced the antitumoral immune response compared with virotherapy or DC vaccination alone. Triggering of the antitumoral immune response was largely 
A

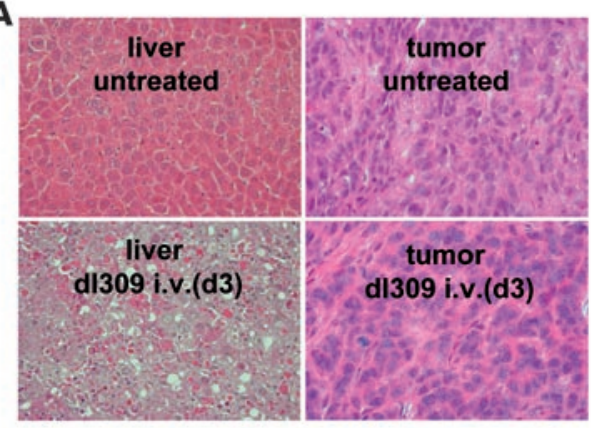

D

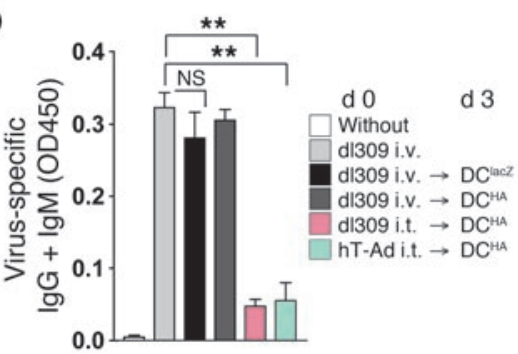

B

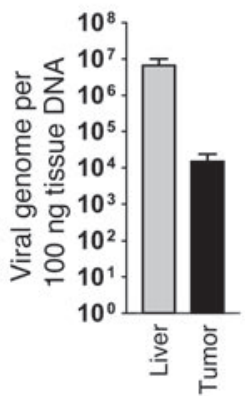

E
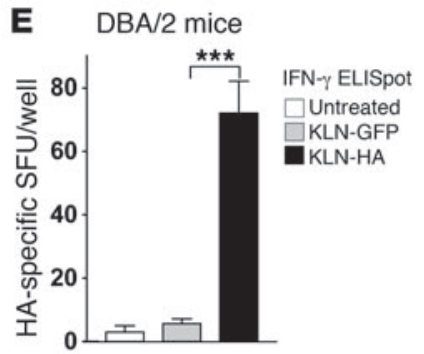

C IFN- $\gamma$ ELISpot

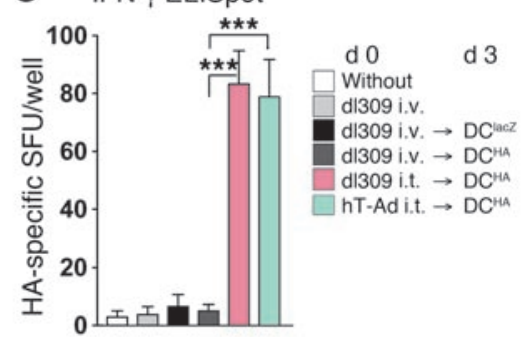

\section{Figure 3}

ODC requires intratumoral inflammation and antigen presence in the tumor tissue. (A) DBA/2 mice bearing KLN-HA tumors were i.v. injected with $1 \times 10^{9}$ infectious particles of dl309. 3 days after treatment, mice were sacrificed, livers and tumors were explanted, and virus-induced tumor inflammation was investigated on H\&E-stained sections. Shown are representative sections of 3 mice per group. Original magnification, $\times 200$ (all panels). (B) Viral DNA load of liver and tumor tissue was quantified on day 3 after i.v. injection of virus by hexon qPCR. (C) On day 3 after i.v. dl309, $\mathrm{KLN}-\mathrm{HA}$ tumor-bearing mice were treated with HA- or lacZ-pulsed DCs and compared with i.t. injection of dl309 prior to vaccination. Infection with hTert-Ad i.t. followed by DC vaccination was performed as ODC reference. After 14 days, mice were sacrificed, and harvested splenocytes were subjected to ELISpot analyses to determine the HA-specific response ( $n=3$ per group, 2 experiments). (D) Serum from 3 animals per group was analyzed for virus-specific Igs by ELISA. (E) DBA/2 mice with s.c. KLN-GFP or KLN-HA tumors received ODC with HA peptide-pulsed DCs. The same experiment was performed in corresponding C57BL/6 CMT-GFP and CMT-OVA mice, who received ODC using hTert-Ad and OVA-pulsed DCs. 14 days after initial treatment, splenocytes were subjected to ELISpot analysis to determine the respective HA- and OVA-specific responses ( $n=3$ per group, experiments repeated twice with similar results). ${ }^{\star \star} P<0.01 ;{ }^{\star \star \star} P<0.001$.

dependent on replication competence of the virus, since injection of rdAd led to a substantially lower frequency of antitumoral CD8 ${ }^{+}$ $\mathrm{T}$ cells. As an additional control, we performed ODC using DCs pulsed with an irrelevant antigen (lacZ). This treatment did not prime a HA-specific $T$ cell response (Figure 2A), confirming the relevance of tumor-directed DC vaccination in ODC. Notably, ODC did not significantly change the $T$ cell response against the viral hexon protein (data not shown). However, investigations on the development of virus-specific Igs revealed a strong inhibition of virus-specific Abs. Comparable to the enhanced antitumoral $T$ cell immune response after ODC, both presentation of the specific TAA by DCs and intratumoral replication of viral DNA were mandatory for the observable decline of the virus-specific humoral immune response (Figure 2B).

Viral infections frequently elicit IgG2a-dominated humoral immune responses, since the Th1 phenotype of virally activated CD4 cells preferentially induces an IgG2a isotype switch $(24,25)$. We investigated whether the observed decrease in antiviral Igs also influences Ig class switching. Therefore, we analyzed the virus-specific IgG2a and IgG1 isotypes, which are characteristic for Th1 and Th2 immune responses, respectively (Figure 2C). In contrast to other treatment groups, ODC inhibited IgG2a production and significantly altered the ratio of IgG1/IgG2a (Figure 2D), indicative of humoral immune responses shifting toward a Th2 phenotype. The shift to Th2-characteristic Ig isotypes was dependent on the use of the specific TAA for pulsing DCs and intratumoral replication of viral DNA. Since there is evidence that IFN-mediated, Th1-directed isotype switching is a prerequisite for effective production of neutralizing Abs (nAbs) against Ads (26), we performed nAb assays. Consistent with our previous findings, we observed a significant reduction of virus-neutralizing activity in the serum of mice subjected to ODC compared with other treatments (Figure 2E).

Induction of virus/tumor immune interference is dependent on virusinduced inflammation of the primary tumor, but the therapentic effect is also conferred to uninfected lung colonies. Our data were suggestive of substantial interference of virus-specific humoral and tumorspecific cellular immunity by ODC. Next, we addressed whether virus-induced inflammation outside the target tumor tissue is also capable of eliciting this immune interference. We performed experiments with i.v. infection of animals with dl309, an E3-deleted Ad5-WT variant resembling hTert-Ad that lacks the tumor-selective control of replication. Injection of dl309 i.v. led to severe hepatitis with a maximum of intrahepatic lymphocyte infiltration on day 3 after infection (Figure 3A). Although systemic application of dl309 led to detectable infection of s.c. tumors, the intratumoral viral load was insufficient to cause apparent lytic destruction of the tumor tissue and lymphocyte infiltration (Figure 3, A and B). In contrast to our results with ODC, we observed neither enhanced antitumoral cellular immunity nor impaired virus-specific humoral immune responses after systemic delivery of dl309 followed by 
A

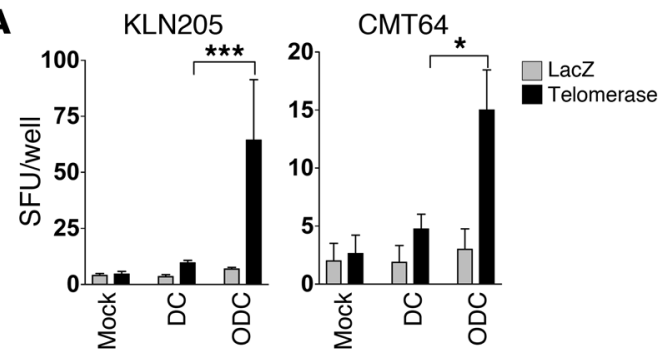

B
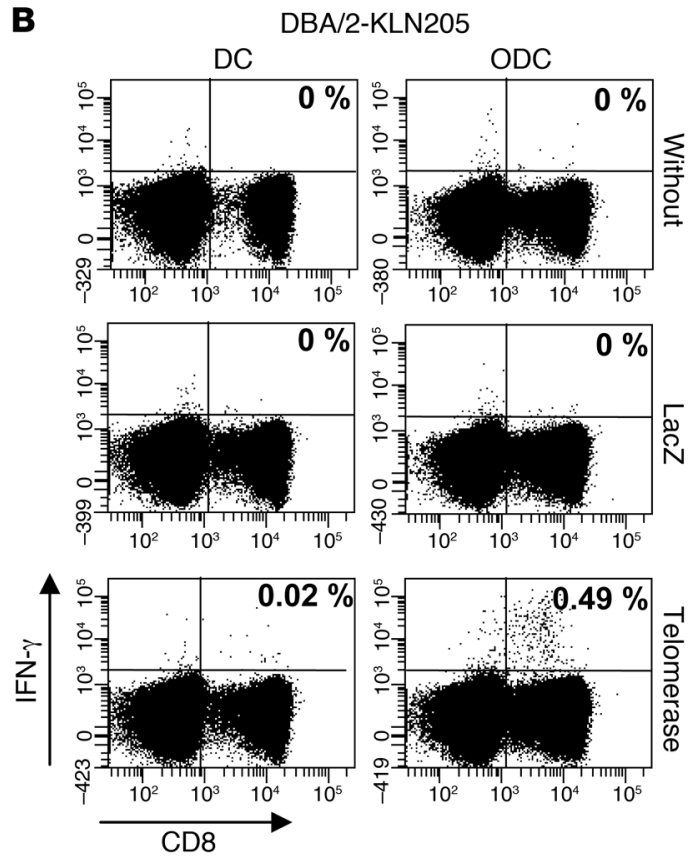

DC vaccination on day 3 (Figure 3, C and D). These observations indicate that inflammation outside the specific target tissue does not result in immune interference.

To address the underlying mechanisms of ODC, we investigated whether the antigen used to prepare the DC vaccine must be part of the viral tumor inflammation, or whether viral tumor-inflammation per se might be sufficient to trigger DC-mediated expansion of $\mathrm{T}$ cells. To this end, we used mice bearing s.c. KLN-HA tumors and KLN tumors expressing GFP as irrelevant antigen (KLN-GFP). All groups received ODC with HA-pulsed DCs. Results of ELISpot analyses showed no immune response against HA in mice bearing KLN-GFP instead of KLN-HA tumors. A significantly elevated $\mathrm{CD} 8^{+} \mathrm{T}$ cell response against $\mathrm{HA}$ after ODC was only detectable when tumors actually expressed HA as TAA (Figure 3E). These results were confirmed in a second model using OVA as antigen (Figure 3E). Our data showed that the antigen used for DC vaccination must be associated with the inflamed tumor tissue for successful $\mathrm{T}$ cell priming.

As shown in Figure 1, ODC therapy strongly improved antitumoral immune responses and tumor regression after vaccination with tumor lysate-loaded DCs. However, for vaccination of cancer patients in clinical settings, the use of single tumor antigens is more common than the use of whole tumor lysates. To investigate whether viral tumor inflammation also allows for vaccination with a single endogenous TAA, we used corresponding peptides against

\section{Figure 4}

ODC is capable of triggering an antitumoral immune response against endogenous TAAs. (A) To investigate the induction of immune responses against endogenous TAAs by ODC, mice with s.c. KLN205 or CMT64 tumors were vaccinated with telomerase-pulsed DCs during tumor tissue inflammation at day 3 after i.t. hTert-Ad. Splenocytes of treated mice were used for ELISpot analyses. Shown are results for the $\mathrm{H}-2^{\mathrm{d}}-$ and $\mathrm{H}-2^{\mathrm{b}}-$ restricted epitopes of telomerase in the KLN205 and CMT64 models, respectively. (B) Intracellular IFN- $\gamma$ staining of $\mathrm{CD}^{+}$ T cells from the KLN205 model after stimulation with the indicated peptides; representative dot plots are shown. ${ }^{*} P \leq 0.05 ;{ }^{\star \star \star} P<0.001$.

the catalytic subunit of murine telomerase, which was strongly upregulated in both CMT64 and KLN205 cell lines (Supplemental Figure 3). In agreement with the results obtained with DCs pulsed with whole tumor lysate or model antigens, ODC with telomerasepulsed DCs strongly supported the antitumoral immune response against telomerase as an endogenous TAA in the KLN205 (H-2d) and CMT64 (H-2 $\left.{ }^{\mathrm{b}}\right)$ models (Figure 4, A and B).

One key mechanism to explain the effect of ODC in immunotherapy appears to be the effective cross-presentation of TAAs in the context of virus-mediated tumor cell death $(5,6)$. Since TLR ligands are currently used as adjuvants in vaccination strategies, we next investigated whether tumor infection with a replicationcompetent virus is necessary or whether mere TLR activation with specific ligands could be sufficient to allow for effective DC vaccination. First, we assessed cross-presentation of the OVA model antigen by APCs after i.t. injection of TLR ligands or viruses. The cross-presentation of the model antigen was directly determined with Abs that can specifically detect the $\mathrm{H}-2 \mathrm{~K}^{\mathrm{b}}$-bound OVA peptide SIINFEKL. Interestingly, only virus infection, but neither of the TLR ligands, led to significant cross-presentation of SIINFEKL on intratumoral APCs (Figure 5A). Moreover, we could not observe cross-presentation on day 1 and 2 after application of TLR ligands (data not shown). Furthermore, significantly more APCs cross-presented OVA-SIINFEKL after tumor treatment with hTert-Ad compared with rdAd, demonstrating that replication of viral DNA in the tumor tissue is an important prerequisite for effective cross-presentation of TAAs on the surface of APCs. In agreement with these results, we observed marginal support of DC vaccination after TLR activation, and only application of the replication-competent virus led to strongly improved efficacy of DC vaccination in ELISpot and in vivo cytotoxicity assays (Figure 5, B and C).

To dissect the roles of intratumoral viral DNA replication and generation of infectious viral progeny in the modulation of immune responses by ODC, we included a further tumor model (Hepa1-6-OVA), since these cells were completely unable to produce infectious particles (Supplemental Figure 1). In contrast to rdAd, the unselective Ad5-WT and the tumor-selective hTert-Ad induced similar lysis in all investigated tumor cells in vitro and in vivo (data not shown). Interestingly, we observed no significant difference between hTert-Ad and Ad5-WT in ODC (Figure 6), in agreement with our previously described findings that the degree of tumor selectivity of Ad DNA replication only marginally influences antitumoral immune responses, but strongly determines anti-vector immunity and systemic toxicity in mice (27). However, whereas replication of viral DNA was an essential precondition for the efficacy of ODC, as shown in our previous experiments, we observed no differences in the antitumoral immune responses in all tumor models (Figure 6), which indicates that de novo gen- 
A
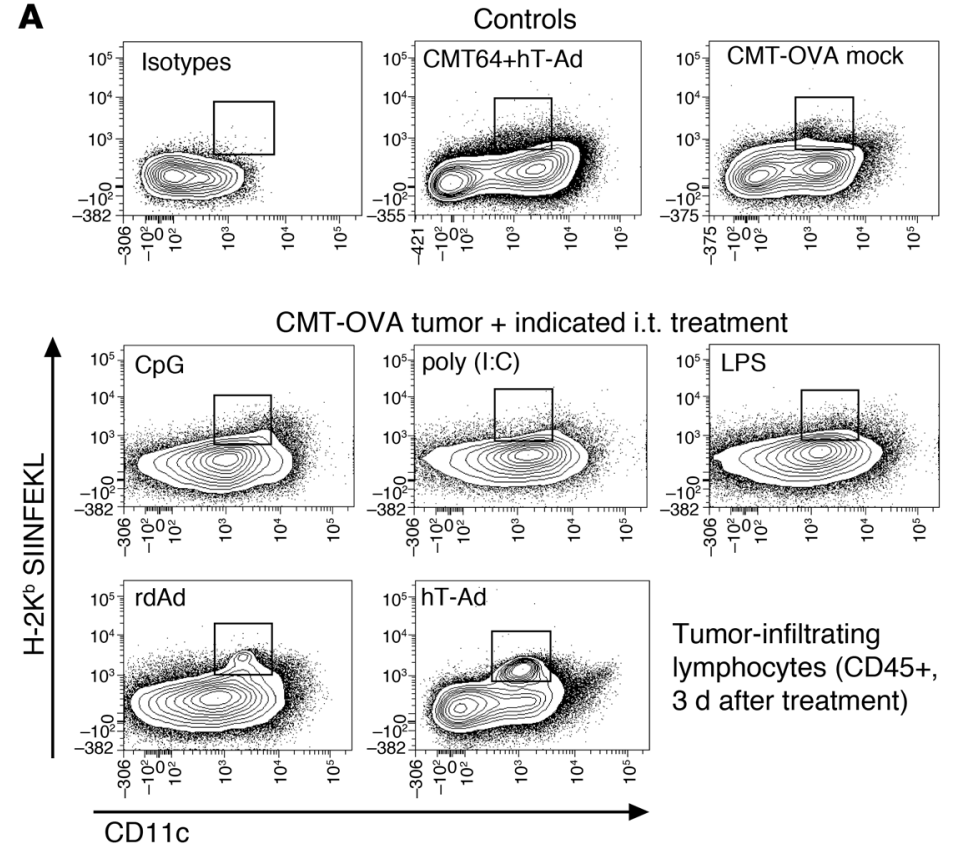

thathant

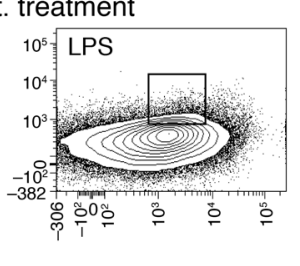

Tumor-infiltrating lymphocytes (CD45+, $3 \mathrm{~d}$ after treatment)

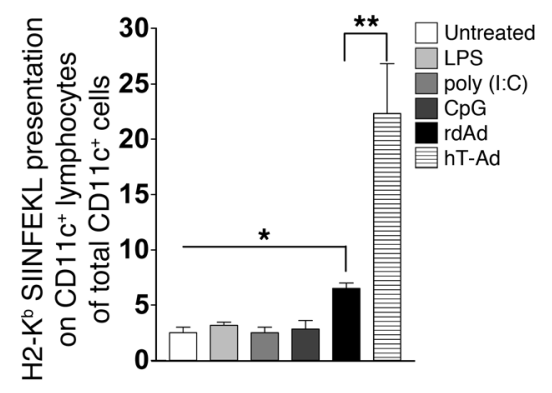

B

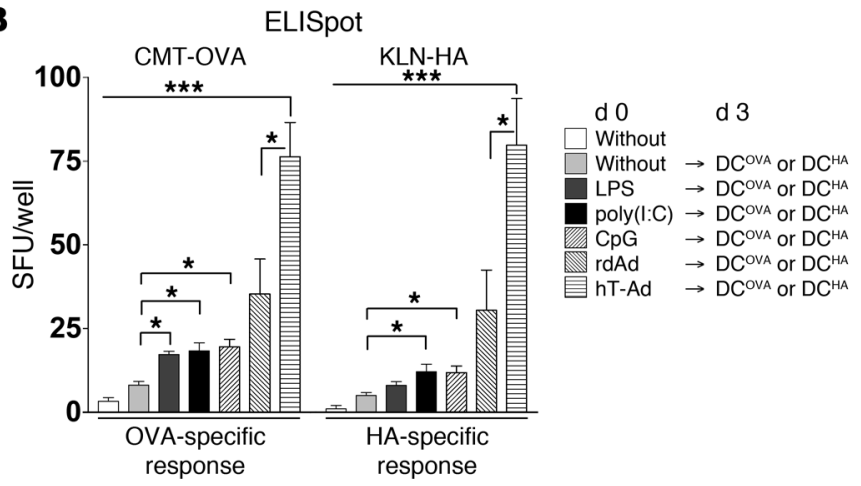

C
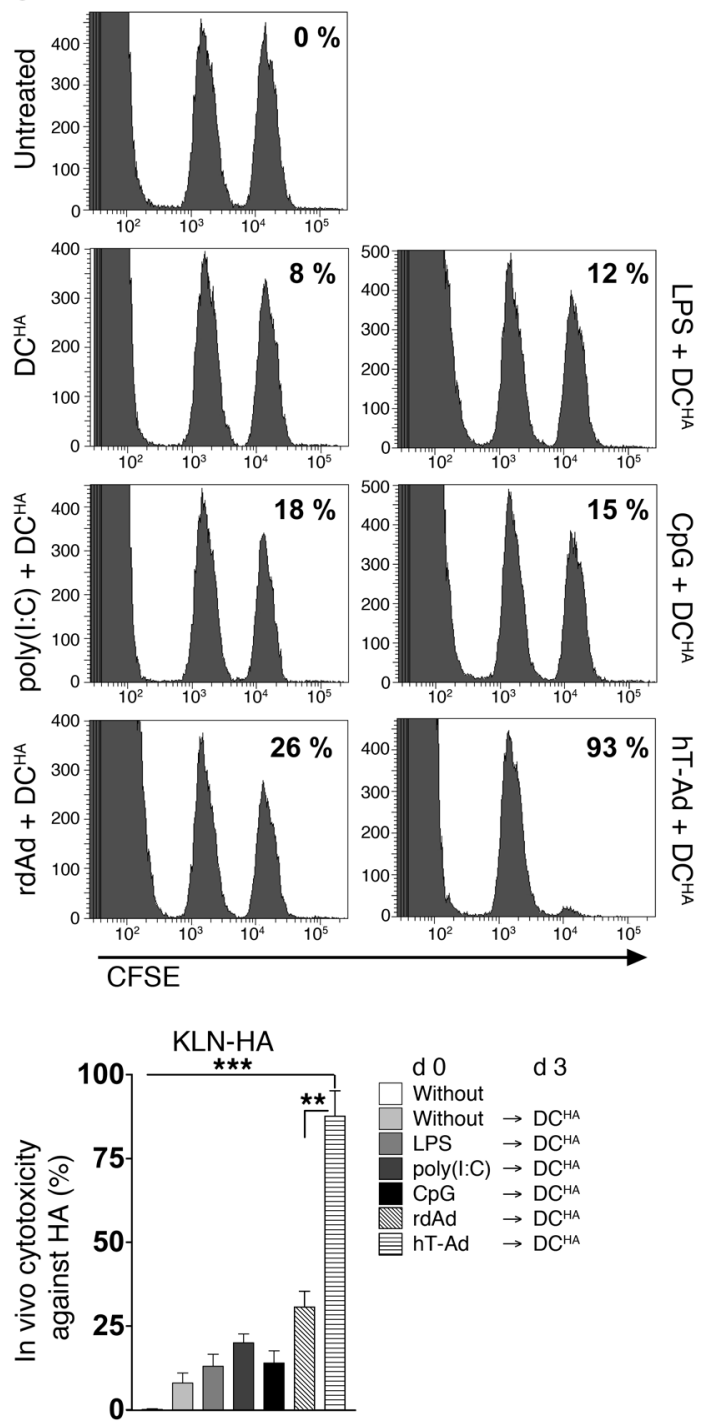

Figure 5

Application of TLR ligands i.t. cannot replace i.t. virus-induced inflammation as a prerequisite for efficient TAA cross-presentation and effective DC vaccination. (A) s.c. CMT-OVA tumors were treated with LPS, poly(I:C), or CpG (see Methods) or with $1 \times 10^{9}$ pfu hTert-Ad or rdAd i.t. 3 days after treatment, and $\mathrm{CD} 45^{+}$cells were prepared from tumor tissue. Cross-presentation of TAAs on APCs was investigated in the CD11 ${ }^{+}$ subpopulation by staining with Ab against SIINFEKL bound to H-2Kb. Maternal CMT64 tumors (lacking OVA expression) infected with hTert-Ad and untreated CMT-OVA tumors served as controls. Shown are representative density plots with the quantification gate for OVA cross-presenting APCs and quantification ( $n=3$ per group, 2 independent experiments). (B) TLR ligands were tested as adjuvants by i.t. injection to support subsequent DC vaccination and were compared with ODC. The experiment was carried out in the CMT-OVA and KLN-HA models. Model antigen-specific T cell responses were analyzed by ELISpot ( $n=4$ per group, 3 independent experiments). (C) T cell responses after i.t. application of TLR ligands and DC vaccination in the KLN-HA model were additionally investigated by in vivo cytotoxicity assays. Shown are representative histograms and quantitative evaluation ( $n=4$ per group, 2 independent experiments). ${ }^{*} P \leq 0.05 ;{ }^{* \star} P<0.01 ;{ }^{* \star \star} P<0.001$. 


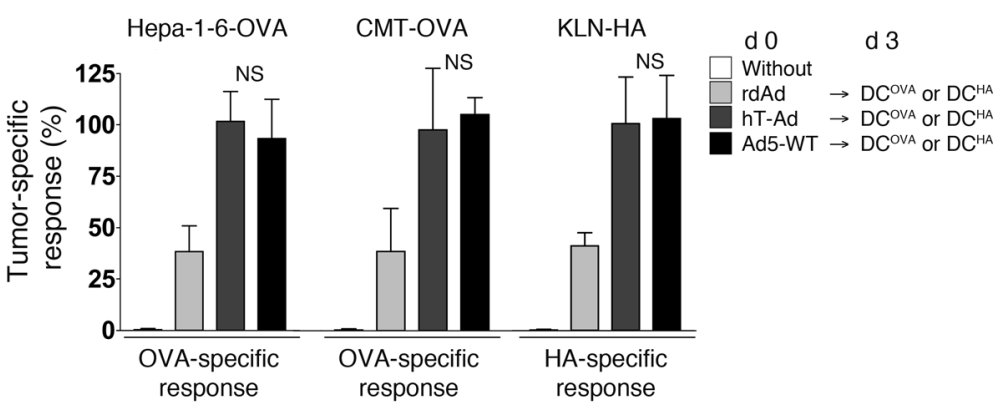

Figure 6

Intratumoral viral DNA replication, but not tumor specificity of the used virus, determines the efficacy of ODC. s.c. KLN-HA, CMT-OVA, and Hepa1-6-OVA tumors were injected with $1 \times 10^{9}$ infectious particles of hTert-Ad (tumor-specific replication) or Ad5-WT (unspecific replication). After 3 days, DC vaccination against the corresponding model antigen was performed. Untreated mice and rdAd treatment served as controls. After 14 days, ELISpot analyses were performed. Shown is tumor antigen-specific response; antigen-specific responses in the respective hTert-Ad groups were defined as $100 \%$ ( $n=4$ per group, 2 independent experiments).

eration of infectious particles within the tumor appears to play a negligible role for the improvement of DC vaccination during viral tumor inflammation.

Since the antitumoral immune response elicited by virus/tumor immune interference was dependent on viral tumor inflammation, we then investigated whether immunotherapy by ODC is also therapeutically effective for treatment of lung colonies that are inaccessible for effective viral injections. Disseminated lung colonies were induced by i.v. injection of KLN-HA cells prior to induction of an antitumoral immune response by subjecting s.c. KLN-HA tumors to ODC. This model allows for investigation of whether ODC-mediated immune responses are capable of reducing tumor burden, which is not a direct subject of virus-mediated tumor cell death. The traces of viral DNA found in tumor-bearing lungs did not differ significantly from those in tumor-free lungs after s.c. tumor treatment with ODC (Figure 7A), confirming the absence of substantial viral infection and DNA replication in lung colonies. Next, we investigated the therapeutic effect of ODC in this model. Compared with untreated controls, we observed smaller tumor lesions and a lower number of lung colonies in the group of animals treated with 2 subsequent virus injections or 2 subse-

\section{Figure 7}

Immune responses induced by DC vaccination during viral tumor inflammation lead to effective elimination of uninfected lung colonies. (A) KLN-HA tumors were grown s.c. on the flanks of DBA/2 mice. At the same time, lung colonies were established by i.v. injection of KLN-HA cells. 14 days later, mice were treated with hTert-Ad i.t. Mice bearing s.c. tumor but lacking the lung colonies were also infected as control. After 3 days, s.c. tumors, lung colonies (after separation from adjacent lung tissue), and lung tissue from lung tumor-free control mice were harvested for DNA preparation. Viral genomes were quantified by qPCR ( $n=3$ per group). (B) In mice with established s.c. KLN-HA tumors, lung colonies were induced 3 days before initial treatment. Mice were then treated with hTert-Ad i.t. followed by ODC compared with mice receiving 2 subsequent virus treatments or $D C$ vaccinations. After 4 weeks, lungs were harvested, and the extent of tumor burden was histologically examined. Original magnification, $x 4$. Tumor area was calculated by computer-based analysis ( $n=8$ per group, 2 independent experiments). ${ }^{\star *} P<0.01$. quent DC vaccinations, respectively. The significant therapeutic effect on lung colonies after single treatments compared with corresponding treatments of s.c. tumors (Figure 1C) is most likely the result of their smaller size and vulnerability that make lung colonies more prone to immune clearance after treatment. However, in line with our results described above, a significantly improved therapeutic effect was observed in the ODC group: only in this group were the majority of lungs from treated animals free of tumor colonies (Figure 7B).

Therapentic efficacy of $O D C$ relies on the presence of Tregs. First, to analyze the contribution of different cell subsets in ODC, we used Abs to ablate myeloid cells as well as $\mathrm{T}$ cell subpopulations. The antitumoral immune response was almost completely inhibited by $\mathrm{CD} 8 \mathrm{Ab}$ and, to a lesser degree, by Gr1 and CD4 Abs (Supplemental Figure 4), which demonstrated that ODC depends on a complex immune response dominated by $\mathrm{CD}^{+} \mathrm{T}$ cells. It has been shown that immune responses after viral infection or tumor vaccination are frequently counterbalanced by Tregs and that CD25 Ab-mediated depletion of Tregs enhances antiviral and antitumoral immune responses $(16,28)$. Therefore, we also investigated whether Tregs are involved in the regulation of antitumoral immune response after ODC. In agreement with other reports (29), CD25 Ab-mediated depletion of Tregs enhanced both antiviral and antitumoral immune responses after 2 subsequent viral applications (Figure 8A). However, eradication of Tregs by application of CD25 Ab resulted in significantly
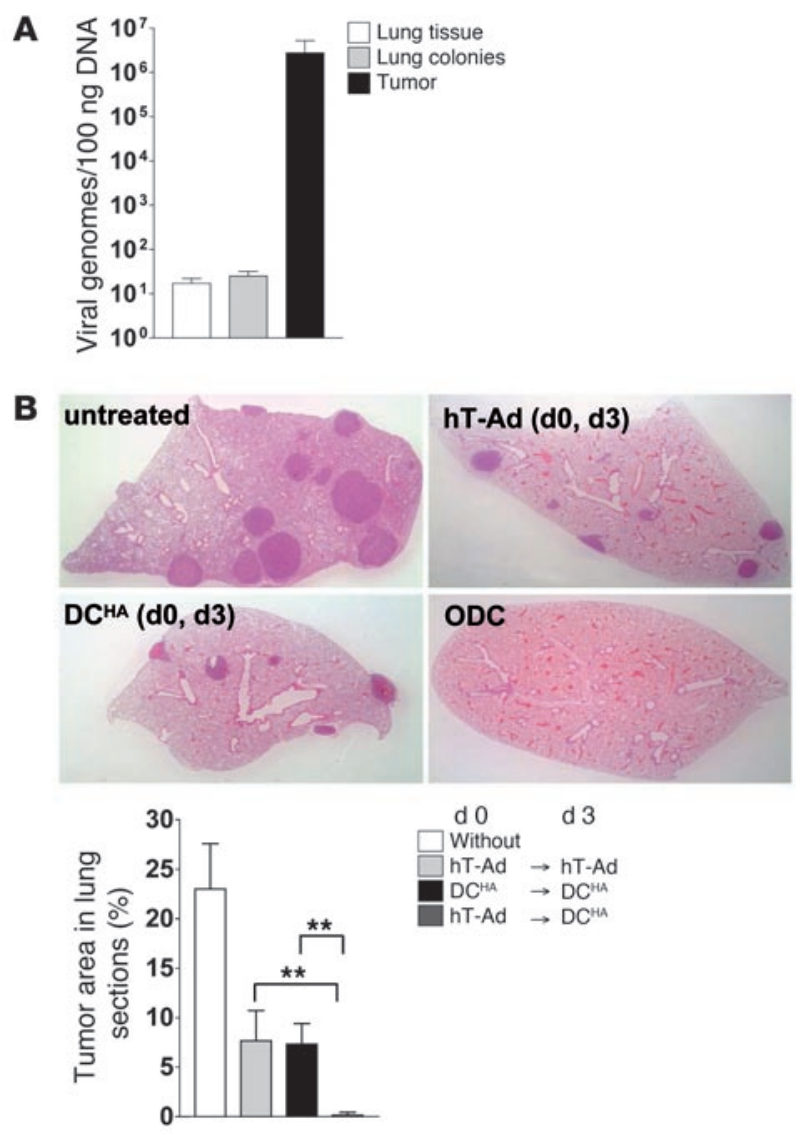

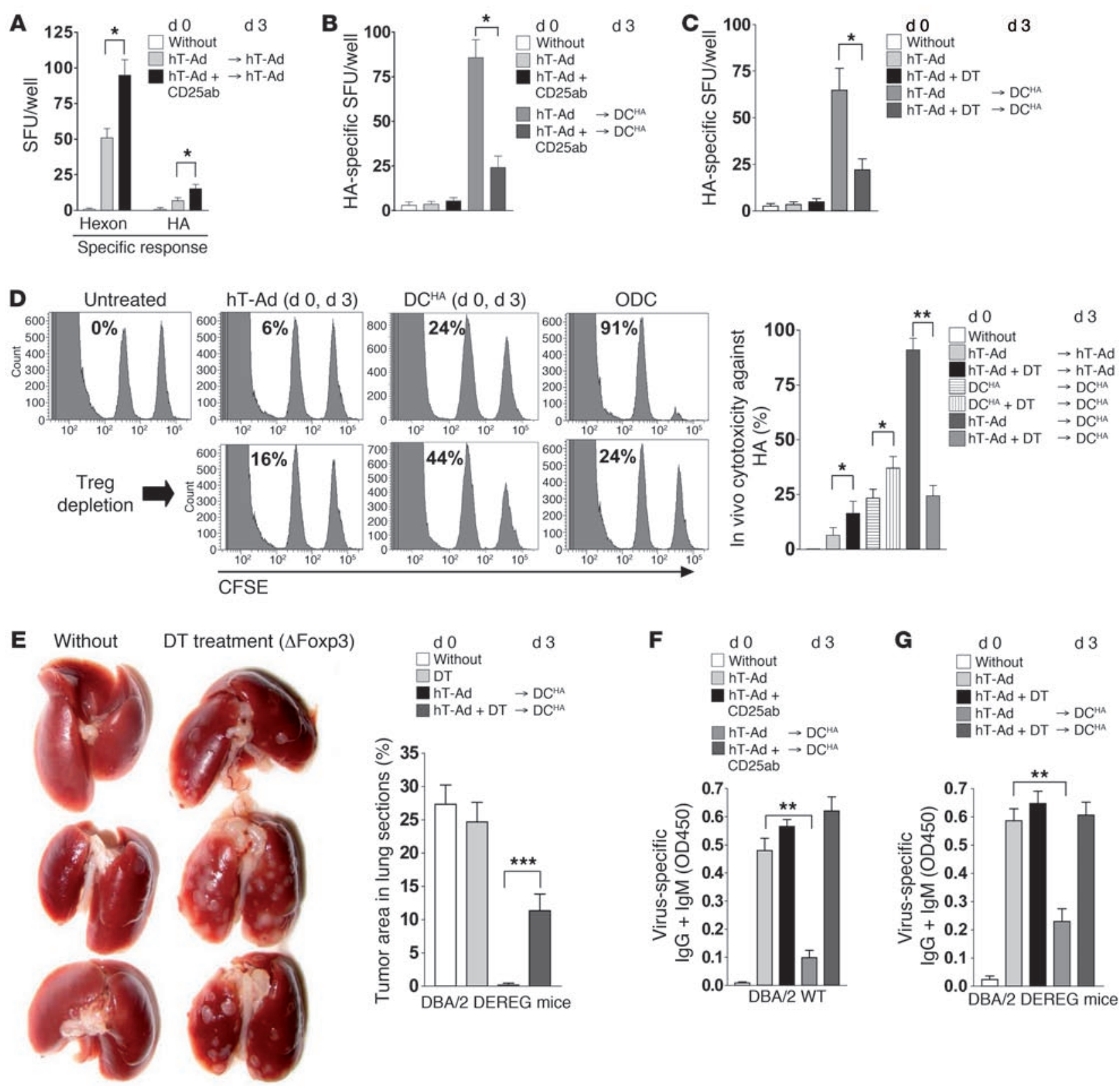

\section{Figure 8}

Potent cytotoxic immune response against TAA after ODC depends on the presence of Tregs. (A) KLN-HA tumor-bearing mice received i.t. virus twice over 3 days. Tregs were depleted by CD25-depleting Abs i.v. 2 days prior to each treatment. 2 weeks later, mice were sacrificed, and splenocytes were prepared and subjected to ELISpot analysis of virus- and tumor-specific immune responses ( $n=4$ per group). (B) Mice received hTert-Ad i.t. and HA-pulsed DC vaccination on day 3, and were Treg depleted by CD25 Ab. i.t. virus or Treg depletion only were additional controls. 14 days after the first treatment, splenocytes were subjected to ELISpot analysis of HA-specific response $(n=5$ per group, 2 independent experiments). (C) Experiment in B was repeated in DEREG mice using DT to deplete Tregs. (D) In vivo cytotoxicity assay of the induced antitumoral immune response. Mice were treated as in Figure 7B, with or without DT. Shown are representative histograms (left peak, lacZ-pulsed cells; right peak, HA-pulsed cells) and quantification of cytotoxicity ( $n=5$ per group, 3 independent experiments). (E) KLNHA tumors and lung colonies were established in DEREG mice as in Figure 7B, and ODC was done. Groups received DT as indicated. After 3 weeks, lungs were inspected for lung colonies (representative images of 5 per group, experiment repeated twice). Tumor area in lung tissue sections was also determined as in Figure 7B. (F and $\mathbf{G}$ ) Serum samples of mice from $\mathbf{B}$ and $\mathbf{C}$ were analyzed for virus-specific Abs by ELISA (IgG and IgM isotypes). ${ }^{\star} P \leq 0.05 ;{ }^{* \star} P<0.01$.

reduced antitumoral immune responses after ODC (Figure 8B). Although application of CD25 Ab is a frequently used method for Treg depletion, this method does not exclusively target Tregs, but also targets activated $\mathrm{T}$ cells that may express CD25 during viral infection. To confirm our results, we additionally used a transgenic mouse model for depletion of Tregs that express a diphtheriatoxin (DT) receptor-GFP fusion protein under control of the foxp3 gene locus (DEREG mice; ref. 30). Similar to our observations after appli- 
A

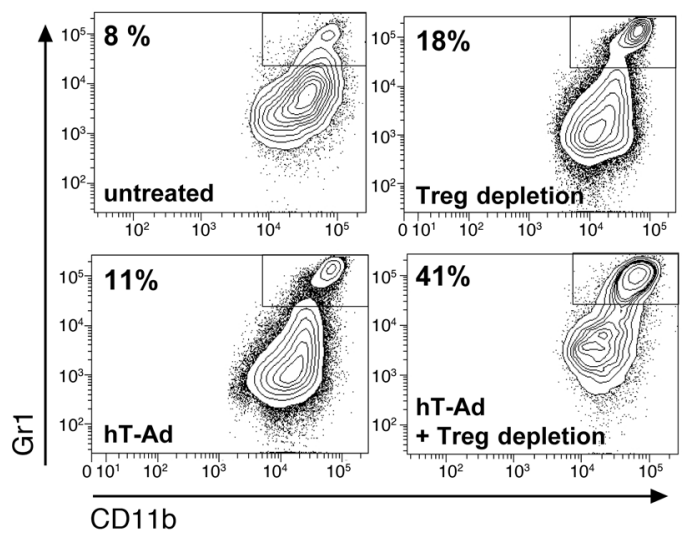

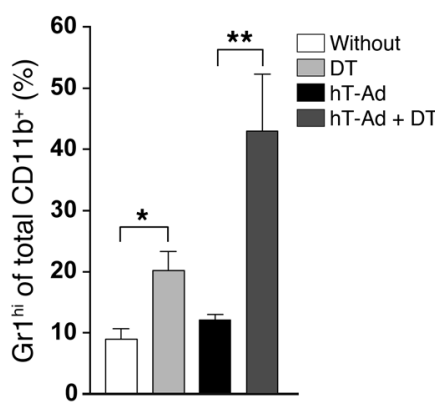

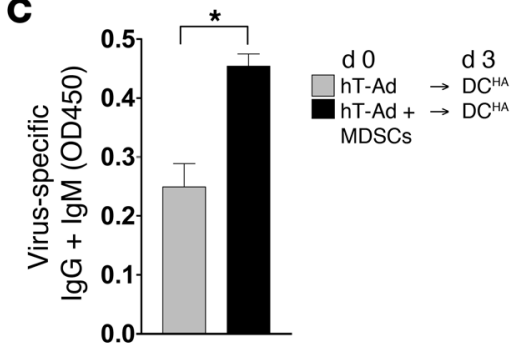

B

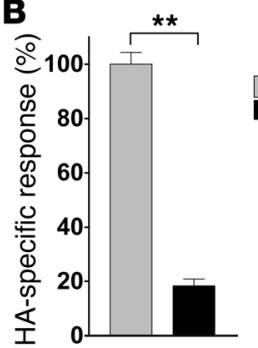

$\begin{array}{lll}\mathrm{d} 0 & \mathrm{~d} 3\end{array}$

$\begin{array}{ll}\square \mathrm{hT}-\mathrm{Ad} & \rightarrow \mathrm{DC}^{\mathrm{HA}} \\ \mathrm{hT}-\mathrm{Ad}+\rightarrow \mathrm{DC}^{\mathrm{HA}}\end{array}$ MDSCs

Figure 9

After i.t. virus infection, vigorous tumor inflammation due to Treg depletion leads to compensatory induction of MDSCs that inhibits the effect of DC vaccination. (A) Treg-depleted, KLN205 tumor-bearing DEREG mice received hTert-Ad i.t. Untreated animals, Treg-depleted animals without viral infection, and hTert-Ad-treated animals without Treg depletion were included as controls. Tumor-infiltrating lymphocytes were isolated and identified by FACS analysis by CD45.2 expression. These cells were gated on CD11b and Gr1. Shown are representative density plots of CD11 $\mathrm{b}^{+}$cells and quantification ( $n=4$ per group, 2 independent experiments). (B) To determine whether MDSCs are responsible for suppression of antitumor immunity by ODC, CD11b+Gr1hiLy6G+ MDSCs were isolated from virus-treated and Treg-depleted donors and adoptively transferred in recipient mice 1 day prior to application of DCs in the context of ODC. Treated mice without adoptive transfer served as positive control $(n=5$ per group, 3 independent experiments). (C) Serum samples of mice were analyzed for virus-specific Abs (IgG and IgM isotypes) by ELISA. ${ }^{\star} P \leq 0.05 ;{ }^{\star \star} P<0.01$.

cation of CD25 Ab in WT animals, depletion of Foxp $3^{+}$Tregs by DT in DEREG mice abrogated the induction of antitumoral immunity by ODC (Figure 8C). We further investigated the effect of ODC and the role of Tregs on the cytotoxic tumor immune response. Results of in vivo cytotoxicity assays demonstrated that depletion of Foxp $3^{+}$ Tregs significantly enhanced the cytotoxic $T$ cell response against HA after virus application or DC vaccination (Figure 8D). However, Treg depletion in DEREG mice nearly abrogated the potent CD8 antitumoral cytotoxicity after ODC (Figure 8D). Consistently, depletion of Tregs did not prevent the outgrowth of lung colonies, but strongly inhibited the therapeutic effect of ODC (Figure 8E). Finally, we investigated whether Tregs are also required to inhibit production of virus-specific Igs after ODC. Depletion of Tregs with CD25 Ab in WT mice or by DT in DEREG mice completely rescued the production of virus-specific Igs after ODC (Figure 8, F and G), which suggests an essential role of Tregs in virus/tumor immune interference.

Abrogation of virus/tumor immune interference by Treg depletion is mediated by $C D 11 b^{+} G r 1^{b i} M D S C$ s. It appears counterintuitive that depletion of Tregs significantly enhances antitumoral immune responses after i.t. virus injection or DC vaccination, but nearly abrogates the virus/tumor immune interference after ODC. We hypothesized that Treg depletion during viral tumor infection may result in an imbalanced vigorous inflammation within the tumor tissue that may provoke potent counterregulations and in turn inhibit the effect of DC vaccination. To investigate the underlying immunological mechanisms, we analyzed cytokine expression patterns of tumor-associated lymphocytes at the time point of DC applications during ODC (day 3 after viral delivery). Depletion of Tregs did not markedly change the expression of cytokines in uninfected tumor tissue (data not shown). In contrast, in tumors with viral infection, depletion of Tregs enhanced the Th1-characteristic and IFN- $\gamma$-dependent chemokines IP10/CXCL10 and CXCL11 (31), as well as TNF- $\alpha$ and the TNF- $\alpha$-dependent TIMP-1 (32). In addition to these central inflammatory mediators, we observed enhanced expression of G-CSF, GM-CSF, M-CSF, and IL-6 in the Treg depletion group during viral replication (Supplemental Figure 5). As these cytokines are known as important factors for generation and maturation of MDSC (33), we then investigated infiltration of MDSCs in tumor tissue. Even in uninfected tumors, depletion of Tregs resulted in significantly higher numbers of MDSCs; however, in line with the altered cytokine profile, we observed an even stronger induction of CD $11 b^{+} G r 1^{\text {hi }}$ MDSCs, particularly in virus-infected tumor tissue after Treg depletion (Figure 9A). Actually, no genetic manipulation has yet been reported that exclusively eliminates $\mathrm{CD} 11 \mathrm{~b}^{+} \mathrm{Gr} 1^{\mathrm{hi}}$ MDSCs, but depletion of myeloid cells has been achieved in some animal models by chemotherapy or by application of Abs against Gr1. However, treatment with Gr1 Ab or Gemcitabine chemotherapy could not rescue the antitumoral immune response after Treg depletion in ODC (Supplemental Figure 4 and data not shown), for which the most likely explanation may be the unspecific elimination of important effector cells, such as $\mathrm{Gr}^{+} \mathrm{DCs}$, in these experiments. To investigate whether the compensatory induction of $\mathrm{CD} 11 \mathrm{~b}^{+} \mathrm{Gr} 1^{\text {hi }}$ MDSCs after Treg depletion contributes to the abrogation of virus/tumor immune interference, we adoptively transferred $\mathrm{CD} 11 \mathrm{~b}^{+} \mathrm{Gr} 1^{\text {hi }} \mathrm{Ly} 6 \mathrm{G}^{+}$MDSCs into animals during ODC. Injection of $\mathrm{CD} 11 \mathrm{~b}^{+} \mathrm{Gr} 1^{\text {hi }} \mathrm{Ly} 6 \mathrm{G}^{+}$MDSCs into the tumors prior to DC vaccination strongly inhibited the antitumoral immune response and rescued virus-specific Ig production (Figure 9, $\mathrm{B}$ and $\mathrm{C}$ ), similar to the results observed after Treg depletion.

In summary, Treg depletion combined with ODC resulted in vigorous and imbalanced intratumoral inflammation, which in turn led to a compensatory induction of MDSCs that abrogated virus/tumor immune interference. Consequently, our data suggest that the optimal therapeutic effect of ODC is strictly linked to the naturally balanced virus-induced inflammation of the tumor. 


\section{Discussion}

Currently, DCs are under clinical evaluation in immunotherapy of cancer as promising means to evoke antitumoral $\mathrm{CD}^{+} \mathrm{T}$ cell responses $(34,35)$. An essential precondition for successful cancer DC vaccination is the generation of large numbers of tumor-specific $T$ cells with potent cytotoxic properties. Unfortunately, several studies indicated that tumor-specific immune tolerance mechanisms impede the development of a therapeutically effective antitumoral immunity after vaccination (36). Therefore, new strategies to improve tumor vaccination with DCs are urgently needed.

It has been reported that viral infection is capable of overcoming immune tolerance mechanisms (37). On the other hand, it is now evident that pathogens induce not only cytotoxic $\mathrm{T}$ cells, but also Tregs, acting as a counterbalance to cytotoxic responses and thus preventing severe immunopathology (9). These observations suggest a well-coordinated balance between effector and suppressor mechanisms in pathogen-associated tissue inflammation.

Our study investigated the potential of virotherapy in connection with tumor vaccination apart from mere cytolysis, since there is growing evidence of the immunomodulatory effects of virotherapy. We performed temporally coordinated vaccinations with TAA-pulsed DCs in the context of viral tumor inflammation with the tumor-specific, replication-competent Ad hTert-Ad in order to shift the balance of emerging antiviral responses in favor of tumordirected immune responses. This approach, termed ODC, elicited a potent antitumor immune response. In contrast, attempts to replace the adjuvant effect of intratumoral replication of Ad DNA by i.t. application of different TLR-activating agents did not elicit a comparable tumor-directed immune response. TLR activators are potent adjuvants to support the generation of cytotoxic $\mathrm{T}$ lymphocyte responses against antigens. However, the presence of TLR activators in the tumor microenvironment after i.t. instillation does not necessarily provide sufficient TAAs in close complex with the TLR ligand that can be engulfed and presented by tumorassociated APCs. In line with this hypothesis, we could not detect significant cross-presentation of TAAs after application of TLR activators; additionally, only a slight enhancement of the antitumoral cellular response was observable in our models.

In contrast, viral tumor infection provides all necessary signals for highly effective tissue inflammation, leading to massive induction of tumor cell death involving close association of TAAs with pathogen-associated molecular patterns. Consequently, viral tumor infection leads to very effective engulfment and cross-presentation of TAAs on the surface of APCs. Accordingly, Blander and colleagues have shown that the presence of TLR-activating agents in vacuoles containing the engulfed material is essential for effective antigen presentation (38). The loose association of TAAs and TLR signaling after application of external TLR activators might therefore limit their use as immunomodulatory agents in cancer immunotherapy.

Our results suggest that intratumoral Ad DNA replication is an essential stimulus for triggering a strong antitumoral immune response. In contrast, productive generation of infectious viral progeny within tumor tissue appeared to be dispensable, since ODC-mediated antitumoral immune responses were also induced in tumors wherein Ad DNA was effectively replicating, but no infectious progeny were produced.

Recently, it has been shown that DC vaccination without concomitant inflammation leads to accelerated generation of memory $\mathrm{T}$ cells that can be massively expanded upon a variety of booster immunizations, including viral infections (39). In our models, only DC vaccination at the time of viral tumor inflammation was capable of eliciting a strong antitumoral cytotoxic $\mathrm{T}$ cell response. These initially surprising results are most likely explainable by the principal malfunction of DC vaccination in the presence of a tolerance-mediating, solid tumor mass. In contrast to vaccination against pathogens, our results indicated that $\mathrm{DC}$ vaccinations against TAAs essentially require the abrogation of tumor tolerance that can be effectively addressed with viral tumor inflammation. We showed that DC vaccinations prior to viral tumor inflammation or after viral clearance and tissue recovery did not trigger antitumoral immune responses in our experiments.

In prime-boost regimens of cancer immunotherapy, heterologous viral vectors as vaccine carriers are attractive alternatives to DC vaccination, since viral vectors can at least transiently break tumor-specific tolerance in tumor-bearing hosts. In a prime-boost strategy with a replication-deficient Ad vaccine followed by an oncolytic VSV expressing a TAA as overlapping antigen, Bridle et al. could enhance antitumoral $\mathrm{CD}^{+} \mathrm{T}$ cell responses and were able to turn the immune response against the oncolytic vector into a beneficial response against the tumor (40). In agreement with these results, we also observed after ODC-mediated enhancement of antitumoral $\mathrm{CD}^{+} \mathrm{T}$ cell responses a significant inhibition of virusspecific humoral immune responses accompanied by an apparent shift to a Th2-characteristic Ig isotype. Although the therapeutic impact of the decline of antiviral nAbs in ODC remains unclear, these results confirm an interference of antitumoral cytotoxic $\mathrm{T}$ cell responses with virus-specific humoral immune responses.

Systemic viral infections may cause generalized immunosuppression characterized by a decline of $\mathrm{CD}^{+} \mathrm{T}$ cell responses and/or nonspecific inflammation $(14,41,42)$. In our models, these concerns appeared to be negligible for viral tumor infection in cancer immunotherapy. We observed that controlled intratumoral replication of viral DNA strongly supported cancer immunotherapy and was an essential precondition for therapeutic success.

It is known that Tregs can act as suppressors of effector $\mathrm{T}$ cell functions during viral infections $(14,15,43-45)$. Consistent with this notion, lack of Tregs significantly enhanced immune responses after single DC or viral treatments. However, when Tregs were depleted in ODC-treated mice, the antitumoral response was abrogated, and virus-specific humoral response was restored. In these animals, we observed significant intratumoral induction of $C D 11 b^{+} G r 1^{\text {hi }}$ MDSCs, an observation that can presumably be interpreted as a compensatory counterregulation against the vigorous, virus-mediated inflammation in absence of Tregs. It has been described in a murine model of local HSV infection (17) that Treg depletion leads to vigorous inflammation in the infection-associated local lymph nodes that inhibits the timely and directed migration of immune cells to the infected tissue. In contrast to these findings, we observed that a vigorous inflammation in virus-infected tumors after Treg depletion supported strong expansion of MDSCs. As an approach to unravel their role in ODC, the adoptive transfer of $\mathrm{CD} 11 \mathrm{~b}^{+} \mathrm{Gr} 1^{\mathrm{hi}} \mathrm{Ly}_{6 \mathrm{G}} \mathrm{MDSC}^{+}$into virus-infected tumors prior to $\mathrm{DC}$ vaccination resulted in abrogation of virus/tumor immune interference. This finding confirmed our hypothesis that a lack of Tregs triggers a compensatory induction of MDSCs, which in turn antagonizes the effect of DC vaccination in ODC. It has been recently shown that, in the context of TLR9 activation, Tregs can undergo rapid reprogramming into activated Th cells that play a vital supportive role in priming of $\mathrm{CD}^{+} \mathrm{T}$ cells in response to cross- 
presented antigen (46). Consistent with these results and those of Lund et al. (17), we found further evidence that $\mathrm{CD} 4^{+} \mathrm{CD} 25^{+} \mathrm{Foxp} 3^{+}$ Tregs are not only general suppressors of immune responses, but are also important for their fine adjustment.

Taken together, our present data suggest that the enhanced antitumoral immune response in ODC is dependent not only on the time point of vaccination and intratumoral viral DNA replication, but also on physiologically balanced virus-induced tumor inflammation. These results indicate that tumor-specific, replication-competent viruses are attractive tools to induce potent tumor inflammation as an essential precondition for effective tumor vaccination.

\section{Methods}

Cells. Huh-7 cells were obtained from the Japanese Collection of Research Bioresources. Hepa1-6 cells, HEK293 cells, the NSCLC cell line KLN205, and A549 cells were obtained from ATCC. The SCLC cell line CMT64 was a gift from W. Jefferies (University of British Columbia, Vancouver, British Columbia, Canada). The SCLC cell line H69 was provided by R. GerardySchahn (Hannover Medical School, Hannover, Germany). Stable expression of model antigens in KLN205 and CMT64 was achieved by retroviral transduction using a Moloney murine leukemia virus (MMLV) containing a HA-IRES-EGFP or OVA-IRES-EGFP expression cassette; MMLV containing EGFP served as control vector. After retroviral transduction, EGFP ${ }^{+}$ cells were sorted by FACS to a purity greater than $90 \%$. All cells were maintained in growth medium (DMEM plus Glutamax; Invitrogen) supplemented with $10 \%$ heat-inactivated FCS (Invitrogen), $100 \mathrm{U} / \mathrm{ml}$ penicillin, and $100 \mu \mathrm{g} / \mathrm{ml}$ streptomycin (Seromed) at $37^{\circ} \mathrm{C}$ and $5 \% \mathrm{CO}_{2}$.

Mice. 6- to 8-week-old C57BL/6 and DBA/2 mice were obtained from Charles River. DBA/2 DEREG mice were obtained by crossing Balb/c DEREG mice with DBA/2 WT mice for at least 4 generations. Resulting mice supported s.c. tumor growth of KLN205. All in vivo experiments were conducted according to the German legal requirements (TierSchG) with approval of Hannover Medical School animal facility. We established s.c. tumors of CMT64 and KLN205 or transgenic subtypes by injecting $1 \times 10^{7}$ cells into the flanks of syngeneic mice. Tumors were grown for 10-12 days to a size of 6-7 $\mathrm{mm}$ in diameter prior to initial viral treatment. Lung colonies were induced by i.v. injection of $1 \times 10^{6}$ tumor cells in PBS 3 days before initial treatment. Viral tumor inflammation was induced by a single i.t. injection of $1 \times 10^{9} \mathrm{pfu}$ Ad. To analyze humoral and cellular immune responses, mice were sacrificed on day 14 after viral treatment. Blood samples were drawn from the heart, and serum was prepared and analyzed by ELISA for virus-specific Igs. Spleens were harvested, and splenocytes were subjected to ELISpot analyses. Depicted tumor sizes were measured using a digital caliper. Tumor volume was calculated as $\left(l \times w^{2}\right) / 2$.

For histological monitoring, mice were sacrificed, and tissues samples of lung, liver, or tumor were fixed with $4 \%$ PFA. $4-\mu \mathrm{m}$ paraffin sections were stained with hematoxylin and eosin according to standard methods.

Abs, peptides, and TLR ligands. The following mouse-specific Abs were purchased from eBioscience: anti-CD16/32; anti-CD4 clone GK1.5; antiCD8 clone 53-6.7; anti-IFN- $\gamma$ clone AN-18; anti-IFN- $\gamma$ biotin-conjugated clone R4-6A2; anti-CD25 clone PC61.5; FITC-conjugated anti-CD11b clone M1/70; PerCP-Cy5.5-conjugated anti-CD45.2 clone 104; purified/ APC-conjugated anti-Gr1 clone RB6-8C5; APC-conjugated anti-CD11c clone N418; and PE-conjugated anti-SIINFEKL bound to $\mathrm{H}-2 \mathrm{~K}^{\mathrm{b}}$ clone 25-D1.16. Furthermore, we used HRP-conjugated anti-IgG/IgM (Serotec), HRP-conjugated anti-IgG1 (Zymed), and HRP-conjugated anti-IgG2a (Zymed) for ELISA. All peptides were obtained from Proimmune: HA (IYSTVASSL, H-2K'), hexon (KYSPSNVKI, H-2K') (47), lacZ (TPHPARIGL, $\mathrm{H}-2 \mathrm{~L}^{\mathrm{d}}$; DAPIYTNV, H-2 $\mathrm{K}^{\mathrm{b}}$ ), ovalbumin (SIINFEKL, H-2K $\mathrm{K}^{\mathrm{b}}$ ), and telomerase
(AYQVCGSPL, H-2K ${ }^{\mathrm{d}}$; SLGKYGKL, H-2K ${ }^{\mathrm{b}}$ ) (48). Peptides were dissolved in DMSO to a final concentration of $2 \mu \mathrm{g} / \mu \mathrm{l}$. TLR ligands were purchased from Invivogen. Mice received $2 \mu \mathrm{g}$ LPS, $200 \mu \mathrm{g}$ poly(I:C), or $50 \mu \mathrm{g}$ CpG (ODN1826) i.t. in PBS. Successful onset of inflammation by application of TLR activators was controlled by FACS analysis of $\mathrm{CD} 45^{+}$lymphocytes in digested tumor tissue.

Ad preparation. The telomerase-dependent replicating hTert-Ad, used as standard replication-competent virus in this study, has been described previously (18). Ad particles of hTert-Ad, dl309, Ad-lacZ, and Ad-GFP were prepared in HEK293 and purified by $\mathrm{CsCl}$ banding according to standard methods. Infectious titer was assayed using the Rapid Titer Kit (BD Biosciences), and endotoxin contaminations of viral preparations were determined with the LAL test kit (Chromogenix) following the manufacturer's recommendations. Virus stocks were stored at $-20^{\circ} \mathrm{C}$ in $25 \%$ glycerol, $10 \mathrm{mM}$ Tris- $\mathrm{HCl}$ ( $\mathrm{pH} 7.4$ ), and $1 \mathrm{mM} \mathrm{MgCl}_{2}$. Before use, viral preparations were dialyzed 2 times with physiologic buffer containing $10 \mathrm{mM}$ Tris (pH 8.0), $1 \mathrm{mM} \mathrm{MgCl}_{2}$, and $140 \mathrm{mM} \mathrm{NaCl}$ at $4{ }^{\circ} \mathrm{C}$.

ELISpot/ELISA. To determine IFN- $\gamma$ release by activated splenocytes, ELISpot assays were performed. For this purpose, 96-well Immobilon-P membrane MultiScreen HTS plates (Millipore) were activated with 35\% ethanol for 5 minutes. Plates were washed 3 times with PBS, incubated overnight with anti-mouse IFN- $\gamma$ Ab clone AN-18 $(7.5 \mu \mathrm{g} / \mathrm{ml})$, and blocked with RPMI medium containing $10 \%$ FCS for at least 2 hours. In all experiments, a single-cell suspension of $2.5 \times 10^{5}$ splenocytes/well was plated in $100 \mu \mathrm{l}$ RPMI medium containing 2\% FCS, $100 \mathrm{U} / \mathrm{ml}$ penicillin, and $100 \mu \mathrm{g} / \mathrm{ml}$ streptomycin including $200 \mathrm{ng}$ peptide or $20 \mu \mathrm{l}$ tumor lysate. After incubation overnight at $37^{\circ} \mathrm{C}$ and $5 \% \mathrm{CO}_{2}$, plates were washed and stained with biotinylated anti-IFN- $\gamma$ Ab clone R4-6A2 and subsequently with HRPstreptavidin conjugate. Spots were visualized by using an AEC staining kit (Sigma-Aldrich). Each value was calculated from 8 wells using an ELISpot reader. Antiviral Igs were detected by ELISA as described previously (22).

$n A b$ assay. To assess the Ad-neutralizing capacity of serum from treated mice, $\mathrm{nAb}$ assay was performed. $3 \times 10^{4} 293$ cells were seeded in a 96-well plate and cultured overnight. Serum was heat-treated at $56^{\circ} \mathrm{C}$ for $15 \mathrm{~min}$ utes to inactivate complement, then serially diluted $1: 1$, ranging from 1:12.5 to $1: 1,600$ in a total volume of $100 \mu \mathrm{l}$ in DMEM with $2 \%$ FCS. For the neutralization reaction, MOI 25 of Ad lac $Z$ was added to all serum dilutions and incubated for 1 hour at room temperature, before the samples were added to 293 cells. 24 hours later, medium was aspired, and cells were washed with PBS. Cells were lysed, debris was pelleted, and the supernatant was used to determine $\beta$-gal activity.

In vivo cytotoxic T lymphocyte assay. Antigen-specific cytotoxic $\mathrm{T}$ cell responses were determined using CFSE-labeled donor splenocytes in an in vivo killing assay as previously described (49). A single-cell suspension of syngeneic splenocytes as targets were pulsed with $10 \mu \mathrm{g} / \mathrm{ml}$ antigen peptide for 30 minutes at $37^{\circ} \mathrm{C}$, then labeled with $2 \mu \mathrm{M}$ CFSE (Invitrogen). Splenocytes pulsed with control peptide (lacZ) were labeled with $0.2 \mu \mathrm{M}$ CFSE. Equal amounts of both cell populations were combined, and $2 \times 10^{7}$ cells total per recipient mouse were injected i.v. 18 hours later, mice were sacrificed, spleens were harvested, and cytotoxicity was determined by recovery of labeled cells by FACS analysis using a FACS Canto II (BD). Antigen-specific cytotoxicity was calculated by the $\mathrm{CFSE}^{\mathrm{hi} /} \mathrm{CFSE}^{\mathrm{lo}}$ ratio.

Generation of DCs for vaccination. Bone marrow cells were isolated from femur and tibia of syngeneic donors. After erythrocyte lysis, cells were resuspended in RPMI supplemented with $10 \%$ FCS, 1 mM sodium pyruvate, $1 \mathrm{mM}$ nonessential amino acids (Invitrogen), and $50 \mu \mathrm{M} 2$-mercaptoethanol (Sigma-Aldrich). Cells were seeded in a 6-well plate and supplemented with $750 \mathrm{U} / \mathrm{ml} \mathrm{rmGM-CSF}$ (R\&D Systems). Cells were fed every 2 days using fresh GM-CSF-supplemented medium. On day 6, cells were additionally loaded with $0.5 \mu \mathrm{g} / \mathrm{ml}$ peptide and activated using $1.5 \mu \mathrm{g} / \mathrm{ml}$ poly(I:C) in 
culture medium. Tumor lysate was generated by 2 freeze/thaw cycles of $2 \times 10^{6}$ syngeneic tumor cells $/ \mathrm{ml}$ in PBS. Debris was removed by centrifugation, and $100 \mu \mathrm{l}$ supernatant/well was used to pulse DCs. The next day, DCs were detached with cell suspension buffer (Invitrogen) and enriched to greater than $90 \%$ by CD11c-MACS purification (Miltenyi). For DC vaccination, mice received $5 \times 10^{5}$ antigen-pulsed DCs by s.c. injection.

Treg depletion. Selective depletion of Tregs was achieved by 2 subsequent i.v. injections of $\mathrm{CD} 25 \mathrm{Ab}(50 \mu \mathrm{g} /$ injection $)$. Ab was administered 2 days before viral treatment and 2 days before DC vaccination. Depletion was controlled after the last CD25 Ab treatment by FACS. 1 week after treatment, CD25 $5^{+}$cells of the $\mathrm{CD} 4^{+} \mathrm{T}$ cell pool were absent (>99\%). Additionally, the DEREG mouse model was used (30). To selectively deplete Foxp $3^{+}$ Tregs, $0.5 \mu \mathrm{g}$ DT (Merck) was applied twice to DBA/2 DEREG mice on the day of viral application and on the day prior to DC vaccination.

FACS analysis of tumor-infiltrating MDSCs and DCs. Tumor tissue from treated mice was minced, and tumor-associated lymphocytes were released by adding RPMI containing $200 \mu \mathrm{g} / \mathrm{ml}$ each of collagenase IA, collagenase $\mathrm{IV}$, and hyaluronidase and $50 \mu \mathrm{g} / \mathrm{ml}$ each of DNase and dispase (all from Sigma-Aldrich). After incubation of the samples at $37^{\circ} \mathrm{C}$ for 30 minutes, the suspension was passed through a $40-\mu \mathrm{m}$ cell strainer and subjected to ficoll gradient centrifugation (Pan Biotech). Isolated lymphocytes were washed 3 times, and Fc receptors were blocked by incubation with anti-CD16/32 $\mathrm{Ab}$ for 10 minutes and then stained with following Abs for MDSC-analysis: anti-CD45.2, anti-CD11b, and anti-Gr1. CD45 ${ }^{+}$cells were gated on $\mathrm{CD} 11 \mathrm{~b}$ and Gr1. Different groups were compared by determining the ratio of the $\mathrm{Gr} 1^{\mathrm{hi}} \mathrm{CD} 11 \mathrm{~b}^{+}$MDSC subpopulation to the total CD $11 \mathrm{~b}^{+}$ population. To detect tumor APCs after therapy, tumor-infiltrating lymphocytes were stained with the following Abs: anti-CD45.2, anti-SIINFEKL bound to $\mathrm{H}-2 \mathrm{~K}^{\mathrm{b}}$, and anti-CD11c.

Adoptive transfer of MDSCs. KLN-HA tumor-bearing DBA/2 mice received a single dose of $50 \mu \mathrm{g}$ CD25-depleting Ab followed by i.t. application of hTert-Ad 3 days before MDSC isolation. MDSCs were isolated from splenocytes of DBA/2 mice using an MDSC isolation kit (Miltenyi) following the manufacturer's instructions. In adoptive transfer experiments, $2.5 \times 10^{6}$ $\mathrm{Gr} 1^{\text {hi }} \mathrm{Ly} 6 \mathrm{G}^{+}$MDSCs were injected into the tumor nodule of treated mice on the day prior to DC vaccination.

Detection of viral DNA. All DNA samples were isolated with QIAamp DNAMini-Kit (Qiagen) and adjusted to $20 \mathrm{ng} / \mu \mathrm{l}$. qPCR of $100 \mathrm{ng}$ DNA from cell culture, lung colony tissue, or tumor tissue was used for quantification of viral genomes compared with a standard curve. Real-time qPCR was performed (qPCR Mastermix Plus; Eurogentec) using a hexon detection kit (Eurogentec) on an ABI 7300 (Applied Biosystems). As an internal control, the 18S-Genomic-Control-Kit (Eurogentec) was applied. 40 cycles with 15 seconds at $95^{\circ} \mathrm{C}$ and 60 seconds at $60^{\circ} \mathrm{C}$ after an initial step of $10 \mathrm{~min}$ utes at $95^{\circ} \mathrm{C}$ were performed.

Statistics. Unless otherwise specified, all values are mean \pm SD. Data were statistically analyzed by unpaired, 2 -tailed $t$ test. $P$ values of 0.05 or less were considered statistically significant.

\section{Acknowledgments}

Research was supported by grants from Deutsche Forschungsgemeinschaft (TRR77), Wilhelm-Sander-Stiftung (2009.005.1), and Mildred-Scheel-Stiftung (107860). We acknowledge the assistance of the Cell Sorting Core Facility of the Hannover Medical School, supported in part by Braukmann-Wittenberg-Herz-Stiftung and Deutsche Forschungsgemeinschaft.

Received for publication October 28, 2010, and accepted in revised form April 13, 2011.

Address correspondence to: Stefan Kubicka or Florian Kühnel, Department of Gastroenterology, Hepatology, and Endocrinology, Medical School Hannover, Carl Neuberg Str. 1, 30625 Hannover, Germany. Phone: 49511.532.6766; Fax: 49511.532.4896; E-mail: Kubicka.stefan@mh-hannover.de (S. Kubicka),kuehnel.florian@ mh-hannover.de (F. Kühnel).
1. Gilboa E. DC-based cancer vaccines. J Clin Invest. 2007;117(5):1195-1203.

2. Melief CJ. Cancer immunotherapy by dendritic cells. Immunity. 2008;29(3):372-383.

3. Ilett EJ, Prestwich RJ, Melcher AA. The evolving role of dendritic cells in cancer therapy. Expert Opin Biol Ther. 2010;10(3):369-379.

4. Kawai T, Akira S. Innate immune recognition of viral infection. Nat Immunol. 2006;7(2):131-137.

5. Chen M, Barnfield C, Naslund TI, Fleeton MN, Liljestrom P. MyD88 expression is required for efficient cross-presentation of viral antigens from infected cells. J Virol. 2005;79(5):2964-2972.

6. Schulz O, et al. Toll-like receptor 3 promotes cross-priming to virus-infected cells. Nature. 2005; 433(7028):887-892.

7. Sfriso P, et al. Infections and autoimmunity: the multifaceted relationship. J Lenkoc Biol. 2010; 87(3):385-395.

8. Rouse BT, Sehrawat S. Immunity and immunopathology to viruses: what decides the outcome? Nat Rev Immunol. 2010;10(7):514-526.

9. Robertson SJ, Hasenkrug KJ. The role of virusinduced regulatory $\mathrm{T}$ cells in immunopathology. Springer Semin Immunopathol. 2006;28(1):51-62.

10. Joosten SA, Ottenhoff TH. Human CD4 and CD8 regulatory $\mathrm{T}$ cells in infectious diseases and vaccination. Hum Immunol. 2008;69(11):760-770.

11. Lanteri MC, et al. Tregs control the development of symptomatic West Nile virus infection in humans and mice. J Clin Invest. 2009;119(11):3266-3277.

12. Lee DC, et al. CD25+ natural regulatory $\mathrm{T}$ cells are critical in limiting innate and adaptive immunity and resolving disease following respiratory syncy- tial virus infection. J Virol. 2010;84(17):8790-8798.

13. Filippi CM, Estes EA, Oldham JE, von Herrath MG. Immunoregulatory mechanisms triggered by viral infections protect from type 1 diabetes in mice. J Clin Invest. 2009;119(6):1515-1523.

14. Dittmer $U$, et al. Functional impairment of CD8(+) $T$ cells by regulatory $T$ cells during persistent retroviral infection. Immunity. 2004;20(3):293-303.

15. Myers L, Messer RJ, Carmody AB, Hasenkrug $\mathrm{KJ}$. Tissue-specific abundance of regulatory $\mathrm{T}$ cells correlates with CD8 $+\mathrm{T}$ cell dysfunction and chronic retrovirus loads. J Immunol. 2009; 183(3):1636-1643.

16. Beilharz MW, et al. Timed ablation of regulatory CD4+ $\mathrm{T}$ cells can prevent murine AIDS progression. J Immunol. 2004;172(8):4917-4925.

17. Lund JM, Hsing L, Pham TT, Rudensky AY. Coordination of early protective immunity to viral infection by regulatory T cells. Science. 2008; 320(5880):1220-1224.

18. Wirth T, et al. A telomerase-dependent conditionally replicating adenovirus for selective treatment of cancer. Cancer Res. 2003;63(12):3181-3188.

19. Younghusband HB, Tyndall C, Bellett AJ. Replication and interaction of virus DNA and cellular DNA in mouse cells infected by a human adenovirus. J Gen Virol. 1979;45(2):455-467.

20. Jogler C, Hoffmann D, Theegarten D, Grunwald $\mathrm{T}$, Uberla K, Wildner O. Replication properties of human adenovirus in vivo and in cultures of primary cells from different animal species. J Virol. 2006; 80(7):3549-3558.

21. Hallden G, et al. Novel immunocompetent murine tumor models for the assessment of replication- competent oncolytic adenovirus efficacy. Mol Ther. 2003;8(3):412-424.

22. Ramakrishna E, et al. Antitumoral immune response by recruitment and expansion of dendritic cells in tumors infected with telomerase-dependent oncolytic viruses. Cancer Res. 2009;69(4):1448-1458.

23. Wang Y, et al. E3 gene manipulations affect oncolytic adenovirus activity in immunocompetent tumor models. Nat Biotechnol. 2003;21(11):1328-1335.

24. Snapper CM, Paul WE. Interferon-gamma and B cell stimulatory factor- 1 reciprocally regulate Ig isotype production. Science. 1987;236(4804):944-947.

25. Maloy KJ, et al. CD4(+) T cell subsets during virus infection. Protective capacity depends on effector cytokine secretion and on migratory capability. JExp Med. 2000;191(12):2159-2170.

26. Zhu J, Huang X, Yang Y. Type I IFN signaling on both $\mathrm{B}$ and CD4 $\mathrm{T}$ cells is required for protective antibody response to adenovirus. J Immunol. 2007; 178(6):3505-3510.

27. Gurlevik E, et al. Selectivity of oncolytic viral replication prevents antiviral immune response and toxicity, but does not improve antitumoral immunity. Mol Ther. 2010;18(11):1972-1982.

28. Yu P, et al. Intratumor depletion of CD4+ cells unmasks tumor immunogenicity leading to the rejection of late-stage tumors. J Exp Med. 2005; 201(5):779-791.

29. Diaz RM, et al. Oncolytic immunovirotherapy for melanoma using vesicular stomatitis virus. Cancer Res. 2007;67(6):2840-2848.

30. Lahl K, et al. Selective depletion of Foxp3+ regulatory $\mathrm{T}$ cells induces a scurfy-like disease. J Exp Med. 2007;204(1):57-63. 
31. Bromley SK, Mempel TR, Luster AD. Orchestrating the orchestrators: chemokines in control of T cell traffic. Nat Immunol. 2008;9(9):970-980.

32. Zhang Y, McCluskey K, Fujii K, Wahl LM. Differential regulation of monocyte matrix metalloproteinase and TIMP-1 production by TNF-alpha, granulocyte-macrophage CSF, and IL-1 beta through prostaglandin-dependent and -independent mechanisms. J Immunol. 1998;161(6):3071-3076.

33. Gabrilovich DI, Nagaraj S. Myeloid-derived suppressor cells as regulators of the immune system. Nat Rev Immunol. 2009;9(3):162-174.

34. Van Tendeloo VF, et al. Induction of complete and molecular remissions in acute myeloid leukemia by Wilms' tumor 1 antigen-targeted dendritic cell vaccination. Proc Natl Acad Sci U S A. 2010; 107(31):13824-13829.

35. Ledford $H$. A shot in the arm for cancer vaccines? Nature. 2010;464(7292):1110-1111.

36. Gross S, Walden P. Immunosuppressive mechanisms in human tumors: why we still cannot cure cancer. Immunol Lett. 2008;116(1):7-14.

37. Kurooka M, Kaneda Y. Inactivated Sendai virus particles eradicate tumors by inducing immune responses through blocking regulatory T cells. Can- cer Res. 2007;67(1):227-236.

38. Blander JM, Medzhitov R. Toll-dependent selection of microbial antigens for presentation by dendritic cells. Nature. 2006;440(7085):808-812.

39. Badovinac VP, Messingham KA, Jabbari A, Haring JS, Harty JT. Accelerated CD8+ T-cell memory and prime-boost response after dendritic-cell vaccination. Nat Med. 2005;11(7):748-756.

40. Bridle BW, et al. Potentiating cancer immunotherapy using an oncolytic virus. Mol Ther. 2010; 18(8):1430-1439.

41. Robertson SJ, et al. Suppression of acute anti-friend virus CD8+ T-cell responses by coinfection with lactate dehydrogenase-elevating virus. J Virol. 2008; 82(1):408-418.

42. Schneider-Schaulies S, Dittmer U. Silencing T cells or T-cell silencing: concepts in virus-induced immunosuppression.J Gen Virol. 2006;87(pt 6):1423-1438.

43. Zelinskyy G, Dietze K, Sparwasser T, Dittmer U. Regulatory $\mathrm{T}$ cells suppress antiviral immune responses and increase viral loads during acute infection with a lymphotropic retrovirus. PLOS Pathog. 2009;5(8):e1000406.

44. Zelinskyy G, et al. The regulatory T-cell response during acute retroviral infection is locally defined and controls the magnitude and duration of the virus-specific cytotoxic T-cell response. Blood. 2009;114(15):3199-3207.

45. Vahlenkamp TW, Tompkins MB, Tompkins WA. The role of CD4+CD25+ regulatory $\mathrm{T}$ cells in viral infections. Vet Immunol Immunopathol. 2005; 108(1-2):219-225.

46. Sharma MD, et al. Reprogrammed foxp3(+) regulatory $\mathrm{T}$ cells provide essential help to support crosspresentation and CD8(+) T cell priming in naive mice. Immunity. 2010;33(6):942-954.

47. McKelvey T, Tang A, Bett AJ, Casimiro DR, Chastain $\mathrm{M}$. T-cell response to adenovirus hexon and DNA-binding protein in mice. Gene Ther. 2004; 11(9):791-796

48. Mennuni C, et al. Preventive vaccination with telomerase controls tumor growth in genetically engineered and carcinogen-induced mouse models of cancer. Cancer Res. 2008;68(23):9865-9874.

49. Mueller SN, Jones CM, Smith CM, Heath WR, Carbone FR. Rapid cytotoxic T lymphocyte activation occurs in the draining lymph nodes after cutaneous herpes simplex virus infection as a result of early antigen presentation and not the presence of virus. J Exp Med. 2002;195(5):651-656. 